\title{
Announcement: Increased Length Limit for Rapid Communications and Discontinuation of Brief Reports in Physical Review A
}

Further changes are afoot for the Rapid Communications section of Physical Review A. As announced on May 1, 2012, renewed standards have been introduced for Rapid Communications in order to place more emphasis on publication of the most important results in the field, rather than rapidity of publication per se. This strategy has worked well, and authors have responded favorably. Now we are moving to give authors a more flexible vehicle for the publication of their best work. Starting with the first issue of volume 91, i.e. January 2015, the length limit will be increased from 3500 words (or approximately 4 pages) to 4500 words (or approximately 5 pages). As before, the word count does not include the title, byline, abstract or references. Simultaneously, authors will be expected to make their articles more self-contained with at most sparing use of Supplemental Material for additional tables or data. All necessary material for a thorough understanding of the article and its significance must be included in the main text. With these changes, we expect to provide our readership with a significantly improved level of understandability for reports on the most exciting developments in the field.

Also starting January 1, we will discontinue the Brief Reports section of Physical Review A. This section, which in recent years contained less than five percent of all the papers published in PRA, was intended for the publication of short articles with limited scope that satisfy the usual publication criteria, but do not reach the same level of completeness as a regular article. There was often confusion among authors and referees about how to interpret these criteria. Moreover, since such manuscripts are supposed to be held to the same high standards as all other regular articles, they should not be relegated to a separate section at the end of the journal Table of Contents. We will still publish short papers that satisfy the usual acceptance criteria as regular articles. This change follows the lead of Physical Review B and Physical Review E, which have already discontinued their Brief Reports sections.

Published 11 December 2014

DOI: 10.1103/PhysRevA.90.060001

PACS number(s): 01.10.Cr 